\title{
The Gravito-Chemical Bond and Structures of Hydrocarbons and Water Molecules with Real Magnetic Charges
}

\author{
Robert A. Sizov* \\ Moscow, Russia \\ Email: sizov.robert@gmail.com
}

How to cite this paper: Sizov, R.A. (2021) The Gravito-Chemical Bond and Structures of Hydrocarbons and Water Molecules with Real Magnetic Charges. Open Journal of Physical Chemistry, 11, 220-240. https://doi.org/10.4236/ojpc.2021.114013

Received: August 6, 2021

Accepted: September 27, 2021

Published: September 30, 2021

Copyright $\odot 2021$ by author(s) and Scientific Research Publishing Inc. This work is licensed under the Creative Commons Attribution International License (CC BY 4.0).

http://creativecommons.org/licenses/by/4.0/ (c) (i) Open Access

\begin{abstract}
Experimental and theoretical studies of the author (period: 1968-present) have shown that true sources of the magnetic field are magnetic fundamental particles (magnetic charges), and not moving electrons. The main reason for ignoring real magnetic charges, as well as true antielectrons in physical science is the hard conditions for confinement of these particles in atoms and substances, which is radically different from the confinement of electrons. Magnetic charges together with electric charges form the shells atoms which are electromagnetic, and not electronic. Namely, electromagnetic shells are sources of gravitational field which is a vortex electromagnetic field and described by the vortex $\operatorname{rot}[\boldsymbol{E}-\boldsymbol{H}]$. Depending on the state polarization of vortex vectors $\operatorname{rot}[\boldsymbol{E}-\boldsymbol{H}]$ in compositions of atomic gravitational fields it is subdivided into paragravitational (PGF) and ferrogravitational fields (FGF). The overwhelming number of atoms emits PGF. Between the masses (bodies, atoms, nucleons and others) emitting PGF areas of negative gravitational "Dark Energy" are realized the forces of which press the masses towards each other. Namely, the compression of atoms by the forces of paragravitational "Dark Energy" underlies the chemical bond. The exception here is the ionic bond in ionic crystals. However, all ions have electromagnetic shells that generate the gravitational field. Consequently, ionic bonding is a relatively rare addition to gravito-chemical bond processes. The direct gravito-chemical bond of carbon atoms with hydrogen $\left({ }^{1} \mathrm{H}\right)$ is physically forbidden due to the manifestation of the effect of ferrogravitational levitation between them and the repulsion of atoms from each other. Paradoxically, but all existing ideas about the structural device of hydrocarbons are based on such physically forbidden bonds which, moreover, must be realized through ionic bonds which in reality do not exist. Chemical bonding of carbon and hydrogen atoms to form hydrocarbons
\end{abstract}

${ }^{\star}$ The author is the doctor of physical and mathematical sciences. At present works as individual researcher. 
molecules is possible only if the hydrogen atoms are in the molecular form $\left({ }^{1} \mathrm{H}_{2}\right)$. In the composition of water, within the framework of the chemical formula $\mathrm{H}_{2} \mathrm{O}$, two stable isomorphic molecular structures are formed. The chemical bond in the first structure is similar to the hydrocarbon scenario described above, i.e. in the process of combining paragravitational oxygen with a hydrogen molecule ${ }^{1} \mathrm{H}_{2}$. The second molecular structure in water is formed under conditions of ferropolarization of the gravitational field of oxygen atoms under the influence of FGF of neighboring ${ }^{1} \mathrm{H}$ atoms. In this case, the chemical bond is realized under the conditions of ferropolarization of the vortex vectors $\operatorname{rot}[\boldsymbol{E}-\boldsymbol{H}]$ of the gravitational fields of all atoms in the molecule and the co-directionality of them vectors $\boldsymbol{P}_{f p}$ ferropolarization. The gravito-physical properties of the presented molecular structures in the composition of water make it possible to name them, respectively, as heavy and light clusters.

\section{Keywords}

Magnetic Charges, True Antielectrons, S-Gravitons, Gravitational Field, Ferro- and Paragravitation, Gravitational Levitation, Gravito-Chemical Bond, Hydrocarbon and Water Molecules, Heavy and Light the Water Clusters

\section{Real Magnetic Charges in Atomic Shells, Para- and Ferrogravitation, Ferrogravitational Levitation}

The experimental and theoretical studies carried out by the author (period: 1968present) have shown that the magnetic charges are real structural components of atoms and substance and are the immediate sources of all magnetic fields in Nature Magnetic particles with a negative charge $\left(\mathrm{g}^{-}\right)$, existing in the compositions of atomic shells, received the author's name magnetons, that should not be confused with such a theoretical surrogate as the Bohr magneton. Magnetons in their physical parameters are magnetic analogs of electrons. Together with electrons and magnetons in the compositions of atomic shells, there are their antiparticles, i.e. true antielectrons $\left(\mathrm{e}^{+}\right)$and antimagnetons $\left(\mathrm{g}^{+}\right)$. Magnetons in their physical parameters are magnetic analogs of electrons [1] [2] [3] [4] [5].

Note 1 . The name and concept of the magneton, given above, should not be confused with Bohr's magneton which is a measure of such theoretical surrogate as the magnetic moment of the electron. The author's research showed that the latter surrogate was introduced into physics to replace real magnetic charges that were "buried alive" in physical theory.

Thus, the shells of atoms are electromagnetic, and not purely electronic, as is now commonly believed [6]. The magnitude charges of electric and magnetic particles in atomic shells correspond to the condition $\mathrm{e}=\mathrm{g}$, where $\mathrm{e}$ is the electron charge. In addition to participating in atomic structures magnetic charges populate potential conduction bands in conductors which, under conditions of superconductivity, turn into zones of real conduction of magnetic charges. It is 
important to emphasize that the above-mentioned real magnetic poles (magnetic charges) have nothing in common with the known Dirac monopoles [7] [8], and also do not participate in such physical manifestations as the magnetricity [9]. All these last theoretical constructions to the formation of the magnetic field used electrons and their currents, i.e. they are, in fact, electrified constructions based on Maxwell's vicious electric magnetism [10] [11].

It is important to note that the first person who experimentally investigated real magnetic charges was the remarkable Austrian physicist Felix Ehrenhaft who for more than 40 years (1910-1952) worked with these "elusive" fundamental particles that actually exist in atoms and substance and make up half of all charged particles [12]. Ehrenhaft's experiments were repeated by his numerous followers who confirmed his conclusions regarding the existence of real magnetic charges in substance (see, for example, [13]).

In 1970, regardless of Ehrenhaft, the author of this article came to the problem of real magnetic charges in atoms and substances based on the results of the own neutron diffraction study of the magnetic structures of hexagonal ferrites [14]. The displacements of the so-called magnetic moments of $\mathrm{Fe}^{3+}$ ions from atomic nuclei, discovered in this study, served as the basis for the author's assumption that these moments are theoretical surrogates invented for compelled replacement of magnetic poles (magnetic charges) which really exist in atomic shell. Subsequent experiments of the author fully confirmed his preliminary conclusions (see, [1] [2] [3]).

The true sources of the magnetic field, i.e. real magnetic charges turned out to be unrecognized in physical science due to the hard conditions of their confinement in atoms and substances which are radically different from the confinement of electrons. In addition, very negative role in history with ignoring of real magnetic charges played the vicious Maxwell's concept of electric magnetism (1873) which is the result of the superficial and, exclusively erroneous, impression of the Great Physicist from the famous experience of Oersted [15] [16].

Note 2. Of course, the experimental activity associated with the detection, extraction and investigation of real magnetic charges is a very difficult and time-consuming job. However, this does not mean at all that these particles should be neglected. Moreover, the author's research has shown that experiments with real magnetic charges are very easier and importantly, more accessible than, for example, the "hunting" on tetraquarks with double charm.

The joint orbital currents of electric and magnetic charges in atomic shells are the source of the Gravitational Field (GF) which is a vortex electromagnetic field. The elementary source of the gravitational field is the electromagnetic quasi-particle which received the name S-Graviton ( $\mathrm{S}=$ source). The S-Graviton is a combination of the electric and magnetic dipoles rotating in antiphase at the same atomic orbit. The model representation of the orbital electromagnetic current or S-Graviton can be written in the following form: $\operatorname{rot}\left[\boldsymbol{J}_{e}-\boldsymbol{J}_{g}\right]$, where $\boldsymbol{J}_{e}$ and $\boldsymbol{J}_{g}$ are vectors the density instantaneous orbital currents of electric (e) and magnetic (g) charges. Then the equation process gravitational field formation by means the S-Graviton can be presented in the form: 
$k \operatorname{rot}\left[\boldsymbol{J}_{e}-\boldsymbol{J}_{g}\right]=\operatorname{rot}[\boldsymbol{E}-\boldsymbol{H}]$ (1), where $\boldsymbol{E}$ and $\boldsymbol{H}$ are the vectors of the instantaneous electric and magnetic field strength in the composition of the vortex electromagnetic (gravitational) field, $k$ is the proportionality coefficient. The minus signs indicated in the above equation for the formation of the gravitational field correspond to both the antiphase of the orbital currents of electric and magnetic charges, and the anti-directionality of the vectors of the instantaneous electric and magnetic field strength at each point of the gravitational field. As will be shown below in the article, it is the noted anti-phase rotation of the magnetic and electric dipoles correspond by the condition for minimizing "Dark Energy".

Vector-vortex analogy between magnetic (a) and gravitational (b) of fields is shown in Figure 1. Unlike from vortex magnetic field of every point which is answered one vector of instantaneous strength of $\boldsymbol{H}$, every point of the gravitational field is responsible for two vectors of instantaneous strength of fields $\boldsymbol{E}$ and $\boldsymbol{H}$ equal by value and oriented antiparallel to each other. In addition, Figure 1 shows that the magnetic vortex field, which is determined by the vortex vector $\operatorname{rot} \boldsymbol{H}$ is formed by means of rotating the magnetic dipole. The elementary source gravitational field is S-Graviton which can be defined as the dynamic EM-bidipole, i.e. two related dipoles (electric and magnetic), rotating in opposite phases on one atomic orbit. The classical equations of the processes formation of magnetic and electromagnetic (gravitational) fields also show in Figure 1.

If the polarization of vortex vectors $\operatorname{rot}\left[\boldsymbol{J}_{e}-\boldsymbol{J}_{g}\right]$ of S-Gravitons is realized in the structures of Physical masses (in atoms, nucleons, substance et al.) that is accompanied by the polarization of vortex vectors $\operatorname{rot}[\boldsymbol{E}-\boldsymbol{H}]$, then by analogy with magnetic fields of the ferromagnetics gravitational fields being emitted these masses can be called the ferrogravitational fields (FGF). The gravitational fields formed by the physical masses in the absence of polarization of the marked

(a)

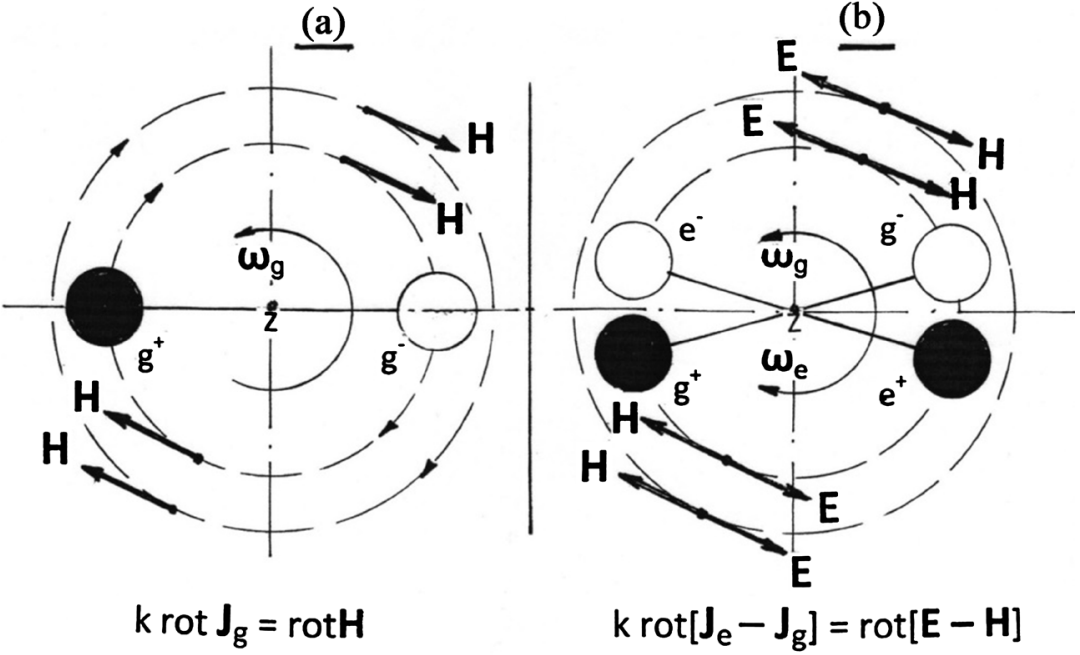

Figure 1. Schemes of mechanisms formation of the vortex spinor fields: magnetic (a) and electromagnetic (gravitational) (b). By white circles on the Figure 1 showed negative charged electric and magnetic spinors: electron $\left(\mathrm{e}^{-}\right)$and magneton $\left(\mathrm{g}^{-}\right)$and black circles are positively charged antispinors corresponding to them with charges $\mathrm{e}^{+}$and $\mathrm{g}^{+}$. 
vortex vectors in their structures are tensor or quasi-scalar fields. And again, by outward analogy with magnetism such fields can to define as Paragravitational Fields (PGF).

It is important to note that the system of two coupled the electric and magnetic dipoles rotating in antiphase in one atomic orbit, is shown in Figure 1(b), and is a real shell of a hydrogen atom (protium). If in the center of Figure 1(b) to place proton, then we get the scheme real atom of protium. It is the electromagnetic shell of protium that represents the S-Graviton which is of the elementary source ferrogravitational field.

The mathematical expression corresponding to the state of the ferrogravitational field has the form $\langle\operatorname{rot}[\boldsymbol{E}-\boldsymbol{H}]\rangle \neq 0$. The gravitational field corresponding to condition $\langle\operatorname{rot}[\boldsymbol{E}-\boldsymbol{H}]\rangle=0$ by analogy with the paramagnetism can be defined as a paragravitational field.

Between the masses (bodies) that emit paragravitational field is implemented generally accepted in physical science the attraction or gravity. Since the overwhelming number of masses in the Universe emit the paragravitational field the processes of "attraction" between them define the famous Law of universal gravitation.

The masses which emit ferrogravitational field repelled from are massessources the paragravitational field, for example, from Earth. This last effect, discovered by the author of the article, is called the effect of Gravitational (Ferrogravinational) Levitation (GL or FGL) [17]. For the purposes of this article, it is important to note that atoms, depending on physical conditions, can emit both PGF and FGF. Between the atomic-sources of PGF and FGF, the GL forces are realized, which repel the atoms from each other, also as the paragravitational field of the Earth pushes out sources of the ferrogravitational field, for example, atoms of light hydrogen (protium). It is the noted effect and the forces of GL that are realized between the atoms that promote the effective decomposition of $\mathrm{CO}_{2}$ and $\mathrm{CO}$ molecules in the author's technology [18].

According to the degree of gravitational stability, atoms are subdivided into gravito-solid and gravito-plastic sources. So, for example, hydrogen atoms (protium) are gravito-solid sources of FGF, and oxygen atoms are gravito-plastic sources which, depending on external circumstances, can emit both PGF and FGF. It is the noted gravito-plasticity of oxygen that is responsible for its high chemical activity.

Since, for more than a hundred years, physical science ignored real magnetic spinor particles (magnetic charges), the effect of gravitational levitation was not discovered and was not used in practice. It is important also to note that currently used renewable energy sources such as wind, sun and even hydroelectric power plants are significantly inferior to the energy potential associated with the Earth's gravitational field.

As part of its experimental research the author has implemented in practice the method of separation of magnetic charges in the pairs $\mathrm{g}^{+}$and $\mathrm{g}^{-}$, as well as 
developed the technology of enrichment of the obtained magneticlly charge. Using the obtained difference of magnetic charged potential, it has implemented the currents of magnetic charges in a lead superconductor. The technique and results of the author's experiments with real magnetic charges and their currents are described in the articles (see, [1] [2] [3]).

\section{Physical Triad of Real World. "Dark Energy" is Unified Source of Power in Nature}

The results of years of research of the real magnetic spinor particles allowed the author to formulate the conception of the World Physical Triad (WPT) according to which the real World consists of three fundamental phases: Matter, Antimatter and Energo-phase (Energo-medium) [19]. Particles Matter are spinors and has charge with negative sign, and of the Antimatter particles are antispinors with positive charge. Particles of Antimatter constitute approximately half all of real spinor particles, i.e. charged particles in real World, and their absence in the physical representations is determined by Physics of hard confinement these particles in structures of substance. The process so-called annihilation of pair: spinor and antispinor, is accompanied by a strong compression of these particles to one another by forces "Dark Energy" ("DE") and, in principle, not lead to the annihilation of the particles.

The Energo-phase (Energo-medium, Power-medium) is of the global medium which executes all force acts on the particles and masses in real World. In the basic (undisturbed) state the Energo-phase is the isotropic superhigh-density gas-like (maybe even quasi-fluid) medium formed by its own fundamental particles referred to as the energions which are spinless and massless. These particles are very small, they move in all directions at speeds close to the speed of light and can be only of the Lefts and Rights what linked with the appropriate direction of their own rotation. General state of the energions within Energo-medium is determined as doubly degenerate. Super-high mobility and not-inertial behavior of the energions allow the particles and masses to move relatively freely in Energo-phase when this medium is in basic (undisturbed) state.

Figure 2 presents a scheme of the World Physical Triad which includes three fundamental World phases: Matter, Antimatter and Energo-medium (Energo-phase). The fundamental particles that constitute Phases of the Triad are indicated on the diagram, i.e. the spinors, antispinors and energions. In Figure 2 marked and such derivatives from phases of Triad as the spinor fields and "Dark Energy". Outside of this scheme remain are condensates consisting of the spinor particles of Matter and Antimatter, called Masses.

According to the Physical Triad Concept all forces direct action on the particles and masses which are implemented in the Energo-phase are the forces of the so-called "Dark Energy" ("DE") which is determined by non-equilibrium states in Energo-medium in the form of oblasts of local pressures created by its own particles-energions. 


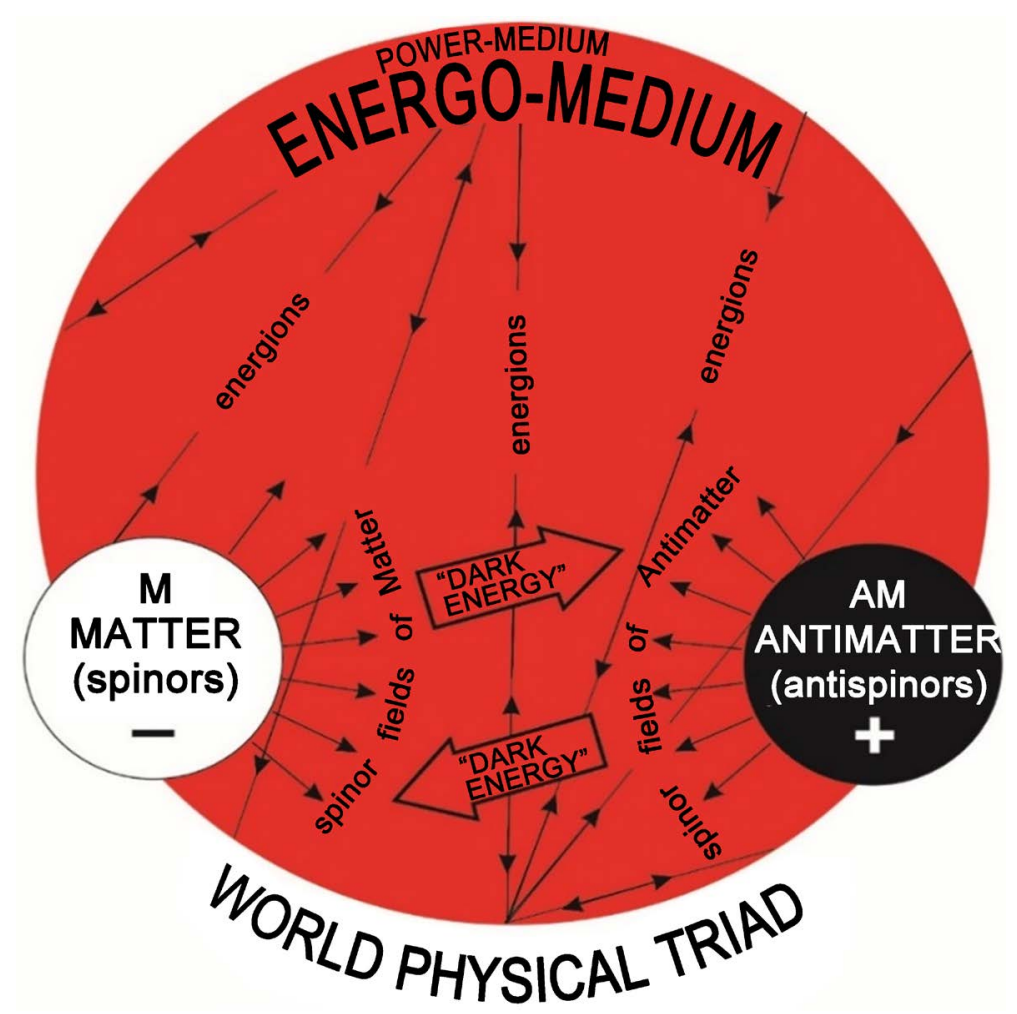

Figure 2. The diagram of the world physical triad.

The formation of "Dark Energy" in Energo-phase is induced by spinor fields, i.e. fields of charged particles. In so doing all of spinor fields including gravitational fields don't have real of power significance. They only play the role of intermediaries exerting influence on state of the Energo-medium and inducing formation of "Dark Energy" in it. Namely "Dark Energy" is real source of forces which are responsible for the dynamics of particles and bodies, as in the scale of the Universe (the movement of galaxies, stars, planets and other objects) so and in the microcosm, for example, in chemical bond formation and dynamics of the spinor particles within in atoms, nucleons, etc.

It is important to understand that it is the energons that are the components of the spinor fields emitted by charged particles, which are fluxes of the energies. Depending on the direction of rotation of energons, spinor fields are subdivided into left-handed (electro-fields) and right-handed (magnetic fields). Spinor fields create energy dissipation in the Energy-medium, which manifests itself in the form of areas as of positive pressure (positive "DE"), so and areas with negative pressure (negative "DE"). The noted pressures in the "Energo-medium" and the forces corresponding to them determine all the observed effects on the particles of Matter and Antimatter and, consequently, on masses (bodies). It is the "DE" induced in the "Energo-medium" by spinor fields is by source of all primary force manifestations in nature. Well-known fundamental forces: gravitational, electromagnetic, strong and weak, are nothing more than a manifestation of the "DE" formations induced in the Energy-medium by the corresponding spinor fields. 
The above statement is easy to accept with respect to the first two forces, which are determined, respectively, by the gravitational "DE" and the common electromagnetic "DE". As for the so-called strong and weak interactions, then, as shown in [20], the corresponding those forces are also the result of "DE" which are induced by the corresponding spinor fields.

Note 3. The Energo-medium can also be called as the Energo and ether, and instead of the ethereal particles known as the Amers in composition of the Physical Triad were been injected particles of the Energions. The introduction of the prefix "Energo" in the composition of the name third component of WPT and its particles is a reflection of the global force significance of this phase in the dynamics of particles and bodies in the real world. The author in his articles showed that namely "Dark Energy" is the global driving force of the Universe.

As noted above in the article, between the Masses (atoms, bodies and other) which emit paragravitational field implemented the so-called the attraction or gravity. It is this last process, which is carried out, most often, by the forces of the gravitational "Dark Energy" is the determining process in the physics of chemical bonding. The Masses which emit the ferrogravtational field are repulsed by from masses-sources of paragravitational field, for example, from Earth, what constitute effect of the Gravitational levitation.

\section{Electromagnetic Shells of Atoms, Gravito-Chemistry and The viciousness of the Theory of Electronic Chemical Bonds}

The discovery and introduction into the basic physical concepts of real magnetic charges, as well as true antielectrons, which together with electrons make up the shells of atoms, allowed the author to develop and propose a new vision of the nature of chemical bonding which is based on the concept of gravito-chemical bonding [21].

The forces responsible for the gravito-chemical bond are the result of the negative gravitational "Dark Energy" that is induced by $\mathrm{PGF}_{\mathrm{s}}$ of atoms in the interatomic regions. It is the so-called paragravitational chemical bond is responsible for the formation of overwhelming number of chemical compounds. The exception here is the ionic bond in ionic crystals. However, all ions have electromagnetic shells that generate a gravitational field. Consequently, ionic bonding is a relatively rare addition to gravito-chemical bond processes. It should be added to this that the ionic bond underlying the formation of molecules hydrocarbons and water is absent in a real physical situation. The ionic model was initiated by the existing theory of electronic chemical bonding, which needs serious correction.

As you know, the formation of chemical compounds, at the moment, is described on the basis of the theory of electronic chemical bonding which arose as the result of a vicious situation in the natural sciences when physicists have "bury alive" the real magnetic charges, as well as true anti-electrons and announced that the shells of atoms are purely electronic. As noted above in the ar- 
ticle, main reason such "burial" this super-rigid conditions of confinement of these particles in the compositions of atoms and substance which are fundamentally different from the confinement of electrons.

In the described situation, which has developed in of the physical-chemical science, the vicious concept of electronic chemical bonding turned out to be the only "life-saving device" using which it was possible all to at least somehow explain. You don't need to be a great thinker to see all the grandiosity and, at the same time, the impracticability of the task that faced the creators and users of the theory of electronic chemical bonding. The problem is that this theory had to explain the complex physicochemical processes occurring in atomic condensates having for this only an electron, that is, one of the four real elementary particles that make up the atomic shells.

Of course, in modern physical theory, magnetic charges and true anti-electrons were replaced, respectively, by such theoretical surrogates as the magnetic moments of electrons and Dirac holes or electronic vacancies [22]. However, this last theoretical "cunning", not only did not resolve the vicious situation in physics, but also created additional complex problems at the interpretation of the effects and manifestations put forward by Nature.

\section{Structural Device of Hydrocarbons and Water Molecules with Real Magnetic Charges}

\subsection{The Electromagnetic Device and Gravito-Physics of Hydrogen Atom (Protium) and Its ${ }^{1} \mathbf{H}_{2}$ Molecule with Real Magnetic Charges}

Hydrogen atoms ${ }^{1} \mathrm{H}$ (protium) which together with carbon and oxygen atoms form, respectively, hydrocarbons and water molecules, largely determine their structure and physical properties. Therefore, it is important to consider in detail the structural device and gravito-physical properties of protium atoms, as well as to clarify their role in the processes of chemical bonding in the noted molecules.

As shown in the author's publication (see, [23]), as well as in Chapter 1 of this article, the elementary source of gravitational field is the electromagnetic quasi-particle which received the name S-Graviton $(\mathrm{S}=$ source). S-Graviton is a coupled pair of electric and magnetic dipole, which rotates in antiphase in the same atomic orbit. The gravitational field created by one S-Graviton is the ferrogravitational field. It is important to note that it is just one S-Graviton that makes up the shell of the protium atom and is the source of its stable FGF. The author's studies have shown that the shells of atoms and nucleons are electromagnetic and consist of atomic and nucleon S-Gravitons. It is the shells of atoms that are the main sources of gravitational fields that are realized in Nature.

In Figure 3 in the framework of structural views of atom of light hydrogen (protium) it atomic shells are shown: currently recognized purely electronic shell (Figure 3(a)) and existing in reality electromagnetic shell (Figure 3(b)). The structure of the hydrogen atom with an electromagnetic shell (Figure 3(b)) which is one S-Graviton is the only physical option that allows creating atomic gravitational 

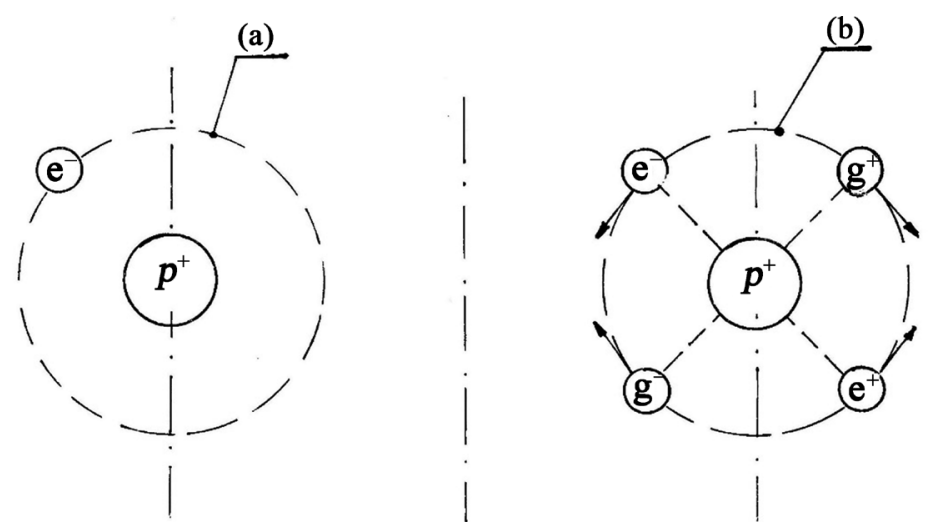

Figure 3. Schematic diagrams of the structure of hydrogen atom (protium): the currently generally accepted atom with a purely electronic shell (a) and the atom existing in reality with an electromagnetic shell (b). At the center of the atoms is the nucleus-proton $\left(p^{+}\right)$ with the charge $\mathrm{e}^{+}$. Arrows for electric and magnetic charges in Figure 3(b) indicate the direction of rotation of the electric and magnetic dipoles in composition of electromagnetic shell of the protium atom.

field. As shown above in the article, the gravitational field of the S-Graviton is the ferrogravitational field.

As noted above in chapter 1, the diagram of device hydrogen atom, shown in Figure $3(\mathrm{~b})$ is reproduced in full in Figure $1(\mathrm{~b})$, if proton $\left(p^{+}\right)$is to place at its center. As follows from Figure 1(b) with proton, the gravitational field, which is formed by the EM-shell of the hydrogen atom is ferrogravitational field. It is important to note that the proton also emits its own gravitational field, which is PGF. However, as shown in the author's publication [24] this proton GF is shielded by the electromagnetic atomic shell of hydrogen atom and does not appear outside of it, i.e. does not participate in chemical bonding processes. Thus, in the processes of gravito-chemical bonding which are realized with the participation of the hydrogen atom ${ }^{1} \mathrm{H}$ the gravitational field emitted only by its electromagnetic shell and which is of stable FGF participates.

The above electromagnetic device of the protium atom and its FGF casts doubt on the manifestation of the ionic bonds in the molecules of hydrocarbons and water. With high of probability, the formation and participation of the ionic state of hydrogen atoms in the formation of the studied molecules is a delusion and has no relation to reality. The author's studies have shown that the chemical bond processes at the formation of hydrocarbon molecules are based on the mechanism of gravito-chemical bonding of carbon atoms with hydrogen which can be realized only if hydrogen is in the composition of ${ }^{1} \mathrm{H}_{2}$ molecules.

Therefore, it is necessary to consider in more detail both the gravito-chemical bond mechanism, which is realized during the formation of a hydrogen molecule, and the state of the molecular gravitational field, which is formed after the combination of two ferrogravitational ${ }^{1} \mathrm{H}$ atoms into the ${ }^{1} \mathrm{H}_{2}$ molecule.

Figure 4 shows the ${ }^{1} \mathrm{H}_{2}$ hydrogen molecule. Dashed concentric lines with arrows in Figure 4 show the $\mathrm{FGF}_{\mathrm{s}}$ of atoms in its composition. In addition, marked in Figure 4 vectors of ferropolarization $\boldsymbol{P}_{f g}$ of gravitational fields of 


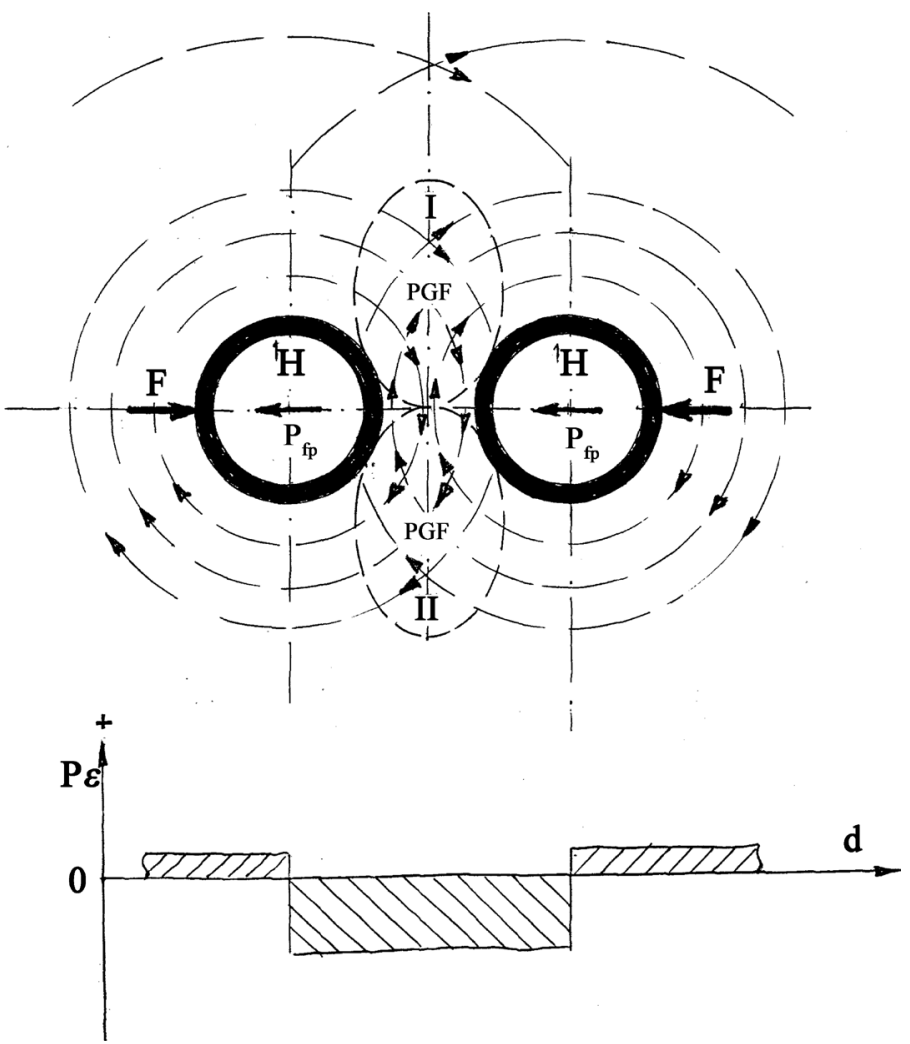

Figure 4. Diagram of the device, the gravitational fields of atoms and forces of the gravito-chemical bond in the hydrogen molecule ${ }^{1} \mathrm{H}_{2}$.

atoms in the composition of the hydrogen molecule.

As noted above, the required condition for the formation of binding forces of hydrogen atoms in the composition of molecule ${ }^{1} \mathrm{H}_{2}$ is the co-directional of vectors of ferropolarization $\boldsymbol{P}_{f g}$ of their $\mathrm{FGF}_{s}$. Only in this case, the forces $\boldsymbol{F}$ of negative "DE" are realized between the hydrogen atoms, pressing the hydrogen atoms in the composition of the hydrogen molecule.

The lower part of Figure 4 shows a diagram of the pressure distribution $P_{\varepsilon}$ of "Dark Energy" forces $F$ which compression of protium atoms in the ${ }^{1} \mathrm{H}_{2}$ molecules.

The particular interest paragravitational regions $\left(\mathrm{PGF}_{\mathrm{s}}\right)$ in the composition gravitational field, which are formed around the hydrogen molecule (highlighted in Figure 4 in the form of two sectorial regions I and II). These areas allow disabling the effect of ferrogravitational levitation between carbon and hydrogen atoms and provide a real chemical bond in hydrocarbon molecules, as well as in one of two molecular clusters in water. The hydrogen molecule in the described situation with it gravitational field can be referred to as the quasi-paragravitational source.

Note 4. In presenting the material of the article, to designate the pressures in the Energo-medium and the vectors of ferropolarization of atomic gravitational fields, the letter $P$, generally accepted for their designation, was used, respectively, in the form of $P_{\varepsilon}$ and $\boldsymbol{P}_{f g}$. 


\subsection{Gravito-Chemical Bond and Structural Device of Molecules of Simplest Hydrocarbons (Alkanes)}

In the concept of Gravitational-Chemical Bond (GCB) which is inescapable in the notions of physics with real magnetic charges and true anti-electrons, direct chemical bonding between a carbon atom and a hydrogen atom (protium) is impossible, in principle, due to the implementation of the effect of gravitational levitation between them. This effect, discovered and investigated by the author, is manifested in the repulsion of the atom-source of FGF from the atom-source of PGF by means of force the positive gravitational "DE" which is formed in the interatomic region (see, [18]). Therefore, the generally accepted schemes of the structural device of hydrocarbon molecules, in which an ion-electronic chemical bond between the $\mathrm{C}$ and ${ }^{1} \mathrm{H}$ atoms assumed and which is depicted in the form of known of hydrocarbon chains is erroneous and cannot be physically realized.

Chemical bond between a carbon atom, which is a stable source of PGF and hydrogen atoms, which are a stable source of FGF can only take place if the latter atoms are combined into the ${ }^{1} \mathrm{H}_{2}$ molecule and act as a single structural complex in the process of chemical bonding with carbon. As shown above in Section 3.1 at hydrogen atoms are combined into a ${ }^{1} \mathrm{H}_{2}$ molecule, in a result of combining atomic $\mathrm{FGF}_{\mathrm{s}}$, two local paragravitational areas are formed around the molecule, which make it possible to exclude the gravito-levitational repulsion between carbon and hydrogen atoms and to carry out the formation of hydrocarbon molecules.

The detailed chemical formulas of the simplest hydrocarbon molecules that meet the conditions of gravito-chemical bond, as well as their structural motifs in the chain representation, have the following form: $\mathrm{H}_{2} \mathrm{CH}_{2}$ и $\mathrm{H}_{2}=\mathrm{C}=\mathrm{H}_{2}$ (methane), $\mathrm{H}_{2} \mathrm{CH}_{2} \mathrm{CH}_{2}$ and $\mathrm{H}_{2}=\mathrm{C}=\mathrm{H}_{2}=\mathrm{C}=\mathrm{H}_{2}$ (ethane), $\mathrm{H}_{2} \mathrm{CH}_{2} \mathrm{CH}_{2} \mathrm{CH}_{2}$ and $\mathrm{H}_{2}=\mathrm{C}=$ $\mathrm{H}_{2}=\mathrm{C}=\mathrm{H}_{2}=\mathrm{C}=\mathrm{H}_{2}$ (propane).

The structural device of other hydrocarbon molecules in the class of alkanes or aliphatic saturated hydrocarbons is determined in a similar way (see Wikipedia "Hydrocarbons"). In this article, we restrict ourselves to considering the structural structure of alkanes. As for other hydrocarbons, such as alkenes, alkynes, dienes and others, their structural arrangement is expected to be presented in the following publications of the author.

Figure 5 shows diagrams of the structural device of simplest hydrocarbon molecules: methane (Figure 5(a)) and ethane (Figure 5(b)), in which the gravito-chemical bond is realized between paragravitational carbon atoms and hydrogen molecules ${ }^{1} \mathrm{H}_{2}$.

The structural unification of carbon atoms and hydrogen molecules in hydrocarbon compositions turned out to be possible exclusively due to the formation of paragravitational areas in the environment of the ${ }^{1} \mathrm{H}_{2}$ molecule, which make it possible to exclude the manifestation of the forces of interatomic gravitational levitation. Paragravitational regions I and II, which are formed in the environment 


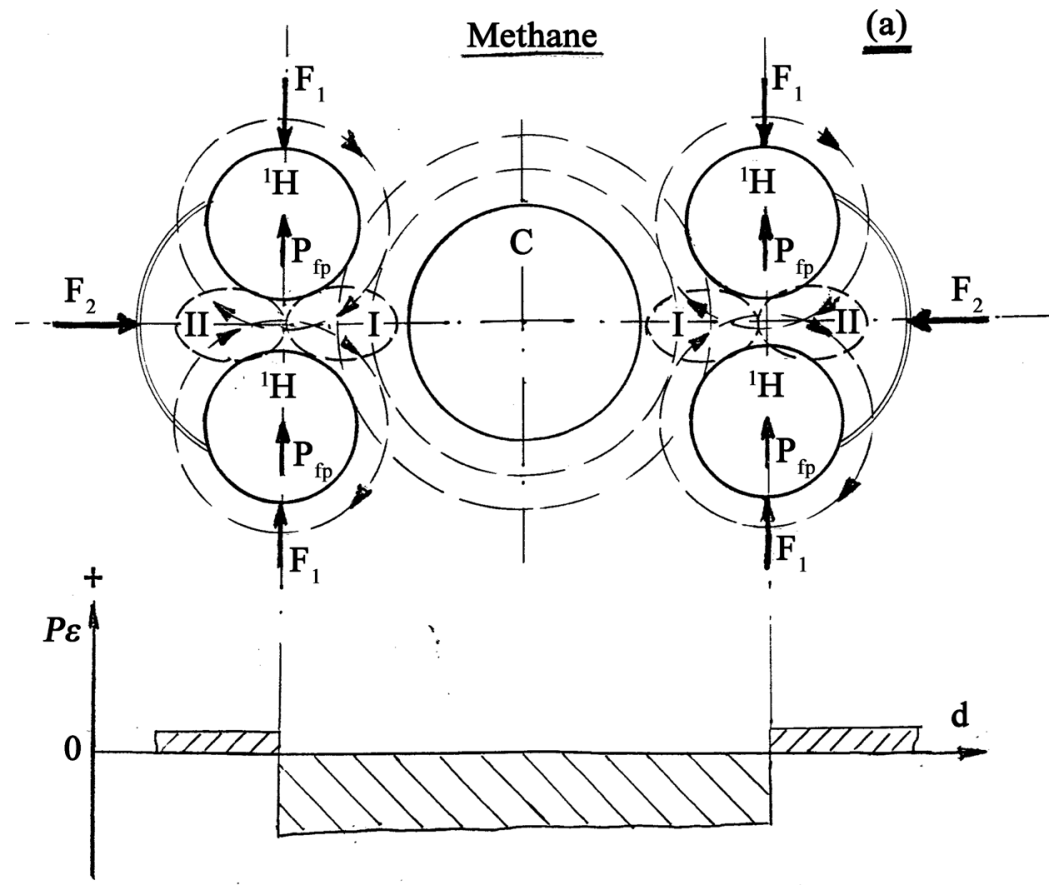

Ethane

(b)

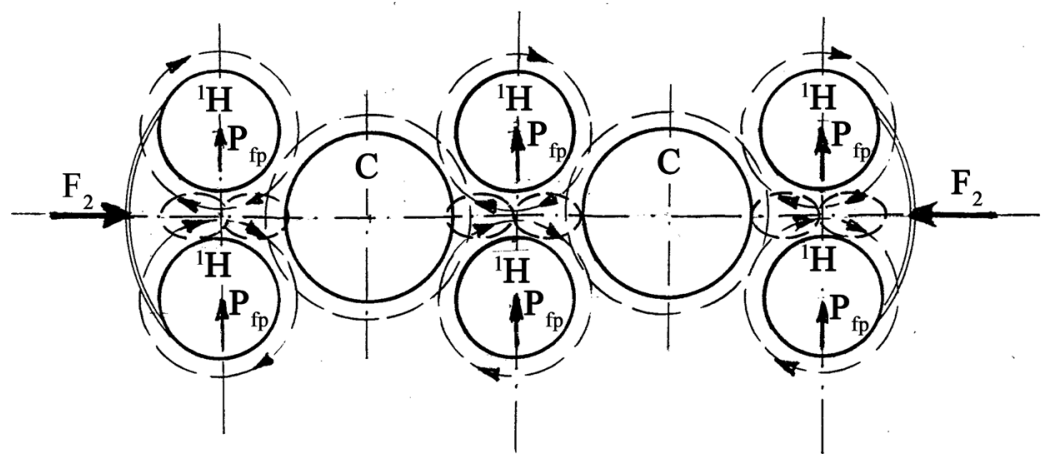

Figure 5. Gravito-chemical bonding and structural device of methane and ethane molecules.

of hydrogen molecules, are highlighted in Figure 5 by intermittent elliptical lines. Shown in Figure 5, as applied to the methane molecule, the distribution of pressures $P_{\varepsilon}$ of "Dark Energy", the forces of which $F_{1}$ and $F_{2}$ pressing the atoms, respectively, into the composition of the ${ }^{1} \mathrm{H}_{2}$ molecule and into the molecule of methane as a whole. The above forces of chemical bonding, which determine the formation of methane molecules, are similarly realized in other hydrocarbons, for example, in ethane and propane.

It is important to emphasize once again that the formation of hydrocarbon molecules can be carried out in the processes of combining carbon with hydrogen, exclusively in the molecular form of the latter. In this situation, when depicting the structural device of hydrocarbons, hydrogen molecules should be considered as components of the main functional group of hydrocarbon molecule. The hydrocarbon chains adopted in the existing structural chemistry, in 
which direct bonds between carbon and atomic hydrogen $\left({ }^{1} \mathrm{H}\right)$ are used, are physically forbidden, and the grandiose structural fantasies generated by them require appropriate correction.

According to the concept of gravito-chemical bond which is realized in hydrocarbons in the framework of the paragravitational scenario described above, it is assumed that ${ }^{1} \mathrm{H}_{2}$ molecules, depending on their location in the molecules, can exhibit valence (with respect to hydrogen) equal to 2 and 4 . The last circumstance should not scare anyone, since we are talking about valence, which refers to a complex of atoms in a hydrogen molecule, and not to an individual atom. Under these conditions, it is possible, for example, to take the PGF oblasts formed by the ${ }^{1} \mathrm{H}_{2}$ molecule (see, Figure 4), as the conditional element of the chemical bond, assigning a valence of 2 to each of them.

It is easy to see that the hydrogen molecules located inside the hydrocarbon chains exhibit a valence equal to 4 , because in this case, the valence of the two PGF zones of the ${ }^{1} \mathrm{H}_{2}$ molecule are involved. As for the hydrogen molecules located on the outskirts of the hydrocarbon chains, then they demonstrate valence equal to 2, because of one of the two zones (the outer PGF zone) of molecule ${ }^{1} \mathrm{H}_{2}$ is free.

As it was repeatedly noted in the article the generally accepted chemical bond of carbon and hydrogen atoms which is usually depicted in the form of $\mathrm{H}-\mathrm{C}-\mathrm{H}$ chains (both horizontal and vertical) is physically forbidden due to the effect of ferrogravitational repulsion between atoms.

It should also be noted that under the conditions of gravito-chemical bonding, the functional groups $\mathrm{CH}_{3}$, which are often used to describe the structure of complex hydrocarbon molecules, are exotic associations, the realization of which is a very rare process. The point is that the marked zones of PGF, which are formed around the ${ }^{1} \mathrm{H}_{2}$ molecule (see, Figure 4) repel the third hydrogen atom, which is the source of FGF. Thus, the ${ }^{1} \mathrm{H}$ atom cannot combine with hydrogen molecule. Such a compound can be realized, exclusively, as a result of an exotic situation when three hydrogen atoms enter into the process of chemical bonding simultaneously and in absolutely identical conditions both in the mutual distances between the atoms and in the direction of the polarization vectors of their FGF $_{\text {s. }}$

\subsection{Molecular Structures (Clusters) in the Composition of Water}

The study of the structural states in the composition of water under the conditions of gravito-chemical binding of hydrogen and oxygen atoms has shown that water is a heterogeneous mass in which two stable molecular structures are realized.

First of all, these are water molecules formed according to the hydrocarbon scenario, in which the paragravity oxygen atom binds to the ${ }^{1} \mathrm{H}_{2}$ molecules, which leads to the formation of so-called paragravitational molecules or clusters with the classical formula $\mathrm{H}_{2} \mathrm{O}$. 
In addition to the so-called paragravitational molecules, the realization in the composition of water of molecules isomorphic to them, which are formed as a result of ferro-polarization of gravitational field of the oxygen atom, is very likely. The point is that, unlike gravitationally stable carbon, oxygen is a gravitoplastic element. Under the influence of ferrogravitational fields of atoms of the nearest environment, in our case these are hydrogen atoms $\left({ }^{1} \mathrm{H}\right)$, the PGF of oxygen atom can be transformed into FGF. In this case, the formation of a gravitochemical bond between oxygen and hydrogen atoms ${ }^{1} \mathrm{H}$ will depend on the conditions of mutual orientation of the ferropolarization vectors $\boldsymbol{P}_{f p}$ of the gravitational fields of atoms in the molecule.

Thus, depending on the state of ferropolarization of oxygen atoms, the presence of hydrogen in the ${ }^{1} \mathrm{H}$ and ${ }^{1} \mathrm{H}_{2}$ states in their environment, as well as on the mutual orientation conditions of the ferropolarization vectors $\boldsymbol{P}_{f p}$ of atomic gravitational fields, possible the following compounds of hydrogen and oxygen in the composition of the water mass.

1) The Paragravitational Cluster (PGC), i.e. the binding of paragravitational oxygen with the ${ }^{1} \mathrm{H}_{2}$ molecule and the formation of the $\mathrm{H}_{2} \mathrm{O}$ molecule, similar to the mechanism of chemical bonding, which is described in Section 3.2 in relation to hydrocarbon molecules $\left({ }^{1} \mathrm{H}_{2}=\mathrm{O}\right)$.

2) The Ferrogravitational Cluster (FGC), i.e. a water molecule in the form of a classical atomic triad $\mathrm{H}-\mathrm{O}-\mathrm{H}$ formed by the binding described above in the conditions of ferrogravitation $\mathrm{GF}_{\mathrm{s}}$ of atoms in the molecule.

3) It is unlikely, but possible that $\mathrm{O}={ }^{1} \mathrm{H}_{2}=\mathrm{O}\left(\mathrm{H}_{2} \mathrm{O}_{2}\right)$, molecules are formed in water with a limited amount of atomic hydrogen and an excess of its molecules in the immediate environment of the paragravitational oxygen atom.

4) $\mathrm{OH}$ complexes, as a result of dissociation of the above-mentioned water molecules under the influence of various external physical factors.

Let us consider the structural features of the above-mentioned basic molecular structures or clusters in the composition of the water mass in more detail.

Figure 6 shows the structural device of isomorphic molecules in the composition of water the so-called: Paragravitational Cluster (PGC) Figure 6(a) and Ferrogravitational Cluster (FGC) Figure 6(b) corresponding to the chemical formula $\mathrm{H}_{2} \mathrm{O}$.

The structural device of PGC is implemented in full compliance with the conditions for the formation of hydrocarbon molecules, discussed above in Section 3.2 of the article. Forces $F_{1}$ and $F_{2}$ in Figure 6(a) determine, respectively, the binding forces of hydrogen atoms in the composition of the ${ }^{1} \mathrm{H}_{2}$ molecule and the binding forces of the constituents of the water molecule. The difference here is that the force $F_{2}$, which presses the ${ }^{1} \mathrm{H}_{2}$ molecule to the oxygen atom, corresponds to the valence value 2, since here only one PGF region of the hydrogen molecule is involved, of the two existing ones $\left(={ }^{1} \mathrm{H}_{2}=\mathrm{O}\right)$. As for hydrocarbons, so the valences related to the hydrogen molecule were corresponded to values of 2 and 4 . 


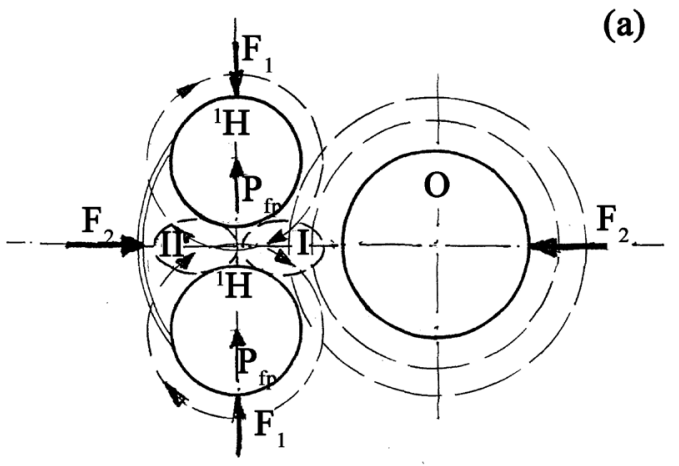

(a)

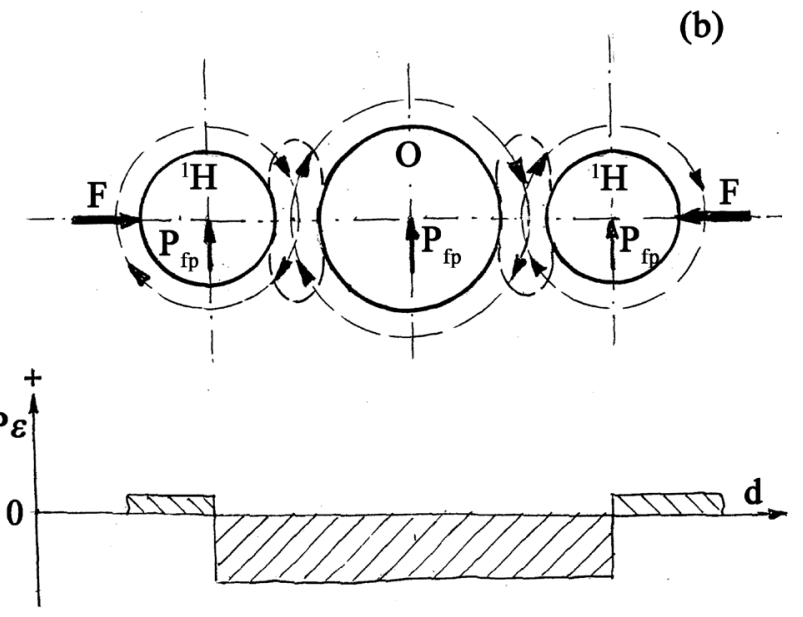

Figure 6. The structural device and forces of gravito-chemical bonding in stable PGC 6(a) and FGC 6(b) clusters that make up the water mass.

As noted above, the possibilities for structuring PGC are determined both by the presence of ${ }^{1} \mathrm{H}_{2}$ molecules in the immediate environment of the oxygen atom, so and by obligatory of the paragravitational state it of the gravitational field.

The second stable structural formation in the composition of water, corresponding to the classical chemical formula $\mathrm{H}_{2} \mathrm{O}$ is referred to as the Ferrogravitational Cluster (FGC). The feature of this cluster is that the gravitational field of oxygen atom, under the influence of the ferrogravitational fields of atomic hydrogen is transformed from paragravitational into ferrogravitational state. In the latter conditions, the binding of the oxygen atom and the ${ }^{1} \mathrm{H}$ atoms possible only if there is the co-directionality of vectors of the ferropolarization $\boldsymbol{P}_{f p}$ of the gravitational fields of all atoms in the molecule. The forces $F$ responsible for the chemical bond in the molecules, as well as the ferropolarization vectors $\boldsymbol{P}_{f p}$ of the gravitational fields of atoms in the FGC composition are shown in Figure 6(b). Figure 6 also shows diagrams corresponding to the pressure zones $P_{\varepsilon}$ of "Dark Energy", the forces of which are responsible for both the chemical bond in the ${ }^{1} \mathrm{H}_{2}$ molecule so and in the composition of the presented aqueous molecular clusters as a whole.

Note 5. The above-mentioned division of molecules or clusters in the composition of water into para- and ferrogravitational formations is conditional. The 
fact is that Nature in $\mathrm{FGC}_{s}$, as and in the gravito-chemistry of hydrocarbons, uses for chemical bonds the PGF regions, which are formed in the interatomic region between the ${ }^{1} \mathrm{H}$ and $\mathrm{O}$ atoms (see Figure 6(b)). In Section 3.1, it was shown that the mechanism of the gravito-chemical bond which corresponds to the conditions for the formation of FGC, is realized also during the formation of ${ }^{1} \mathrm{H}_{2}$ molecules, and it can be called the hydrogen gravito-chemical bond.

It is also important to note that the gravito-physical properties of the presented water clusters suggest significant differences in the interaction of their gravitational fields with the gravitational field of the Earth. So PGC, due to its more significant PGF, are stronger attracted to the Earth than $\mathrm{FGC}_{s}$, and they can be called the heavy clusters. As for $\mathrm{FGC}_{\mathrm{s}}$, because of the ferrogravitational states in the molecule and possible of the gravito-levitation manifestations, they will be weaker attracted to the Earth and they can rightfully be called the light clusters. The gravito-physical properties of the water clusters presented above are based on the real nature of the gravitational field, the manifestations of para-and ferrogravity, as well as the physics effect of gravitational (ferrogravity) levitation discovered and investigated by the author (see, [4] [17]).

In addition to the aforementioned stable water clusters the structures of which correspond to the basic chemical formula of water $\left(\mathrm{H}_{2} \mathrm{O}\right)$, in the composition of liquid water $\mathrm{H}_{2} \mathrm{O}_{2}$ can be realized the states $\mathrm{H}_{2} \mathrm{O}_{2}\left(\mathrm{O}={ }^{1} \mathrm{H}_{2}=\mathrm{O}\right)$. Note that the last structural states, which are formed under conditions of the surplus of ${ }^{1} \mathrm{H}_{2}$ molecules near oxygen atoms with PGF, should be considered, in the author's opinion, as unlikely. The exact ratio of the amount of the described structural states in the composition of liquid water remains to be done. However, there is no doubt that this ratio is not constant and can vary depending on the physical conditions in the state of the water mass.

\section{Discussion}

The study of structural states in the composition of water, carried out by the author, showed the heterogeneity of its composition, postulated by W.C. Röntgen many years ago. The stable isomorphic molecular structures in the composition of the water mass, marked above as PGC and FGC, based on their gravito-physical properties, are defined, respectively, as heavy and light clusters. Note that the above definitions of water clusters (heavy and light) are also accepted in the existing theories of structural states in the composition of water.

It is interesting to compare our results with data from recent experimental studies (2019) of liquid and gaseous water through inelastic X-ray scattering [25]. First of all, the splitting of the resonance inelastic scattering spectrum into two peaks observed in the noted experimental study of liquid water, in all likelihood, testifies in favor of the heterogeneity of water and the existence in its composition of two stable clusters of presented in of our article. As for the similar splitting the resonance into two peaks in the X-ray spectra of scattering by water molecules in the gas phase, according to the author, this indicates in favor of the 
stability of the presented water clusters, which retain their structural identity and in the gas phase.

At the same time, considering the above experimental study and the conclusions made by its authors, it is impossible to abandon the basic idea promoted by the present author that all interpretations of physico-chemical processes in molecular structures that are based on the vicious theory of electronic chemical bond need serious correction. So, for example, is completely erroneous the authors' conclusion of X-ray examination noted above, that the structural associations that exist in the composition of water are "due to the attraction between positively charged hydrogen atoms and negatively charged oxygen atoms". The latter statement is based on the concept of vicious theory of electronic chemical bonding and is erroneous, since water is not an ionic structure, and the formation of the molecules described above in its composition is determined exclusively by interatomic gravito-chemical mechanisms.

Of course, in the composition of pure water, i.e. without any impurities limited ionic states can be realized, which, for example, determine its low electrical conductivity. However, these states cannot lead to the formation of structural clusters presented in the article.

\section{Conclusions}

The tragic situation that has developed in physical science as a result of the vicious ignoring, for almost 150 years, of the true sources of the magnetic field, magnetic charges, turned physics, and with it natural sciences, into "The Kingdom of crooked mirrors". Together with magnetic charges, physicists "buried alive" and such fundamental particles existing in atomic shells are as true antielectrons. Thus, the shells of atoms, which in reality are electromagnetic and include the four fundamental particles described in the article, in the existing physical theory, for more than 100 years, are recognized as purely electronic. At the same time, it is easy to foresee that all interpretations of physical effects and manifestations made on the basis of the existing electronic theory will, to one degree or another, be physically defective. The forced replacement of ignored fundamental particles with the noted in the article theoretical surrogates does not save the tragic situation that has developed in physical science but only drives this "disease" into the dark depths of its theory.

In addition, the author's research has shown that the forces confinement of ignored particles in the structures of substances increases sharply with an increase in the internal energy of the mass (body). Therefore, the existing fascination of world physics with high-energy projects is a serious obstacle to the legalization of magnetic charges and true antielectrons, for the working with which low temperatures, for example, superconductivity conditions, are desirable.

Of course, the model of the electron shell of atoms is of the grand theoretical "puncture", but should be aware that the first place on the harmfulness in physics is necessary to give of C.J. Maxwell and his fallacious electromagnetic con- 
ception [26]. Namely vicious EM-concept of Maxwell, which is called in the publication author as "vicious virus Maxwell's", can associate with the "cancerous" an education in the "body" of physical science, the "metastasis" of which pierce through many directions of physical theory, giving them a false and even mystical significance. Among such directions, may be mentioned, for example, such theoretical constructs as global expand Universe, very big explosion and a big break, the field and the Higgs boson, the representation of the gravitational field in the form of curved four-dimensional space and very many others.

Especially regrettable is the existing status quo theory of gravitational field, which, as the author has shown, is just of the vortex electromagnetic field. For its formation and use in practice, it is not at all need to bend the 4-dimensional spacetime. All that needs to be done for this, so this is to legalize real magnetic charges and learn how to work with them.

Conducting global "chemotherapy" of the modern physical theory with the purpose of eliminating the numerous misconceptions generated by Maxwell's vicious concept, according to the author, is the most urgent task for today. All these need to be done, and as soon as possible, because after 10 - 15 years even robots will laugh at us.

\section{Acknowledgments}

The author expresses deep gratitude to Elena Sizova and Vitaly Sizov, who have helped and supported me for many years, contributing largely to the implementation of my research activities.

\section{Conflicts of Interest}

The author declares no conflicts of interest regarding the publication of this paper.

\section{References}

[1] Sizov, R.A. (2020) The Experiments Detecting of Real Magnetic Charges in Structures of Atoms and Substance. Journal of Modern Physics, 11, 1245-1261. https://doi.org/10.4236/jmp.2020.118078

[2] Sizov, R.A. (2020) Experimental Ferrogravitational Field around Untwisting Closed Superconductor. Journal of Modern Physics, 11, 1807-1826. https://doi.org/10.4236/jmp.2020.1111113

[3] Sizov, R.A. (2021) The Magneto-Motive Force, Currents of Magnetic and Electric Charges in Static Superconductor and Them Vortex Spinor Fields. Journal Current Trend in Physics Research and Applications, 2, 1-10. https://katalystpub.com/jctpra-articles-inpress/

[4] Sizov, R.A. (2015) Real Magnetic Poles (Magnetic Charges) in the Physics of Magnetism, Gravitation and Levitation. Journal of Modern Physics, 6, 1013-1022. https://doi.org/10.4236/jmp.2015.68106

[5] Sizov. R.A. (2001) New Presentation of Nature Magnetism, Gravitation and Nuclear Forces of Bonding. Akademizdat Center "Science", Moscow, 62 p. 
[6] Sizov, R.A. (2016) Electromagnetic Shells of Atoms and the Periodic System of Elements. Journal of Modern Physics, 7, 2374-2397. https://doi.org/10.4236/jmp.2016.716206

[7] Dirac, P.A.M. (1931) Quantized Singularities in the Electromagnetic Field. Proceedings of the Royal Society A, 133, 60-72. https://doi.org/10.1098/rspa.1931.0130

[8] Sizov, R.A. (2018) Real Magnetic Charges as a Negation of the Electric Magnetism Maxwell and Electrified Dirac's Monopole. Physics \& Astronomy International Journal, 2, 17-20.

[9] Bramwell, S.T., Giblin, S.R., Calder, S., Aldus, R., Prabhakaran, D. and Fennell, T. (2009) Measurement of the Charge and Current of Magnetic Monopoles in Spin Ice. Nature, 461, 956-959. https://doi.org/10.1038/nature08500

[10] Maxwell, J.C. (1873) Treatise on Electricity and Magnetism. Vol. 1-2, Clarendon Press, Oxford.

[11] Sizov, R.A. (2019) The Electrical Magnetism of Maxwell (1873) Is the "Crooked Mirror" of Physical Science. Applied Physics Research, 11, 49-55. https://doi.org/10.5539/apr.v11n3p49

[12] Ehrenhaft, F. (1952) On Photophoresis, the True Magnetic Charge and on Helical Motion of Matter in Fields. Acta Physica Austria, 5, 12-29.

[13] Mikhailov, V.F. and Ruzicka, J. (1989) Magnetic Charge in the Experiments by F. Ehrenhaft and Their Modern Development. Acta Physica Universitatis Comenianae, 29, 97-148.

[14] Sizov, R.A. (1971) Delocalization of the Magnetic Moment of $\mathrm{Fe}^{3+}$ Ions in Type Y Hexagonal Ferrite at 293 K. Journal of Experimental and Theoretical Physics, 33, 737-740.

[15] Sizov, R.A. (2017) "Electrical" and "Magnetic" Worlds in Universe. Journal of Modern Physics, 8, 1072-1086. https://doi.org/10.4236/jmp.2017.87069

[16] Sizov, R.A. (2019) The "Naked Electron". Applied Science and Innovative Research, 3, 257-263.

[17] Sizov, R.A. (2015) Real Magnetic Charges in the Substance, Ferrogravitation and Technical Levitation. Journal of Modern Physics, 6, 1591-1601. https://doi.org/10.4236/jmp.2015.611161

[18] Sizov, R.A. (2021) Decomposition $\mathrm{CO}_{2}$ and $\mathrm{CO}$ in Flow of Gases by Means of Technical Ferrogravitational Field. European Journal of Applied Physics, 3, 21-28. https://doi.org/10.24018/ejphysics.2021.3.3.78

[19] Sizov, R.A. (2016) World Physical Triad: Matter, Antimatter and "Dark Energy" in the Processes of Climatic Changes on the Earth. Journal of Modern Physics, 7, 558-572. https://doi.org/10.4236/jmp.2016.76059

[20] Sizov, R.A. (2018) Nuclear Physics with Magnetic Charges. Journal of Modern Physics, 9, 145-171. https://doi.org/10.4236/jmp.2018.92010

[21] Sizov, R.A. (2020) Gravitational Chemical Bond with Real Magnetic Charges and True Antielectrons. Open Journal of Physical Chemistry, 10, 48-67. https://doi.org/10.4236/ojpc.2020.101003

[22] Sizov, R.A. (2015) Dirac's “Holes" Are the True Antielectrons and Real Particles of Antimatter. Journal of Modern Physics, 6, 2280-2289. https://doi.org/10.4236/jmp.2015.615232

[23] Sizov, R.A. (2017) Magnetic and Electric Charges in Physics and Technology of Electromagnetic and Magnetoelectric Induction. Journal of Modern Physics, 8, 964-981. https://doi.org/10.4236/jmp.2017.86061

[24] Sizov, R.A. (2019) Intra-Atomic Gravitational Shielding (Lensing), Nuclear Forces 
and Radioactivity. Journal of Modern Physics, 10, 59-73.

https://doi.org/10.4236/jmp.2019.101006

[25] Vaz da Cruz, V., Gel'mukhanov, F., Eckert, S., Iannuzzi, M., Ertan, E., Pietzsch, A., et al. (2019) Probing Hydrogen Bond Strength in Liquid Water by Resonant Inelastic X-Ray Scattering. Nature Communication, 10, Article No. 1013.

https://doi.org/10.1038/s41467-019-08979-4

[26] Sizov, R.A. (2020) Great Mistake of Great J. C. Maxwell. Applied Science and Innovative Research, 4, 32-38. https://doi.org/10.22158/asir.v4n1p32 